\title{
Laparoscopic procedure compared with open radical hysterectomy with pelvic lymphadenectomy in early cervical cancer: a retrospective study
}

This article was published in the following Dove Press journal:

OncoTargets and Therapy

\author{
Jianfeng Guo* \\ Lu Yang* \\ Jing Cai \\ Linjuan Xu \\ Jie Min \\ Yi Shen \\ Zhoufang Xiong \\ Weihong Dong \\ Vichitra Bunyamanop \\ Zehua Wang
}

Department of Obstetrics and Gynecology, Union Hospital, Tongji Medical College, Huazhong University of Science and Technology, Wuhan 430022, China

*These authors contributed equally to this work
Correspondence: Zehua Wang

Department of Obstetrics and Gynecology, Union Hospital, Tongji Medical College, Huazhong University of Science and Technology, 1277 Jiefang Avenue, Wuhan, 430022, China

Tel +862785351649

Email zehuawang@163.net
Objective: To compare clinical outcomes in laparoscopic and open radical hysterectomy with pelvic lymphadenectomy (LRH) in early cervical cancer without the selection bias.

Methods: One special retrospective study was conducted with more than 400 patients involved in laparoscopic procedure.

Results: Our results suggest that estimated blood loss and transfusion requirements were significantly lower in the LRH group. Postoperative hospital stay was also significantly shorter in the LRH group. Significant difference was found in the number of pelvic lymph nodes retrieved between the LRH and open radical hysterectomy with pelvic lymphadenectomy (ORH) groups. There were no differences in operating time, perioperative complications, progression-free survival, and overall survival between the LRH and ORH groups.

Conclusion: LRH can be considered a safe and effective alternative to conventional open surgery (ORH) for early-stage cervical cancer.

Keywords: early cervical cancer, clinical outcomes, laparoscopic radical hysterectomy, pelvic lymphadenectomy, retrospective study

\section{Introduction}

Among women worldwide, cervical cancer is the second most common cancer and the third leading cause of cancer fatalities. ${ }^{1}$ Two categories, "early stage" and "advanced stage", are currently used for succinctly dividing the staging system of cervical cancer. ${ }^{2}$ For the past one century, open radical hysterectomy with pelvic lymph node dissection $(\mathrm{ORH})$ has been considered as the standard surgical treatment for early-stage cervical cancer. ${ }^{3,4}$ Due to the lengthy period of operation, people gradually found that large volumes of blood loss, transfusion-related complications, and bladder dysfunction were significant morbidity concerns that were associated with ORH. ${ }^{5,6}$ Laparoscopy has become a widely used minimally invasive technique associated with its well-established advantages including lesser blood loss, fewer transfusions required, and a shorter hospital stay. ${ }^{7}$ Since the first laparoscopic hysterectomy was performed to treat benign disease, ${ }^{8}$ laparoscopy has been preferentially used in gynecologic oncology to transition from radical abdominal hysterectomy to radical vaginal hysterectomy. ${ }^{9,10}$ After the first total laparoscopic radical hysterectomy with pelvic lymphadenectomy (LRH) was performed by Nezhat et $\mathrm{al}^{11}$ in 1989 , in the last 2 decades various groups have published their experience about laparoscopic methods, demonstrated the feasibility and safety of the procedure, and suggested that prognosis or survival is not adversely affected by the procedure. ${ }^{12-15}$ Thus, minimally invasive endoscopic surgery has become 
an acceptable alternative for open method procedures. Nevertheless, the questions need to be raised regarding the oncological outcome and the safety of the 2 procedures. There is only 1 randomized controlled trial which had compared the postoperative outcomes between LRH and abdominal radical hysterectomy by enrolling 30 cervical cancer patients in 1 center. ${ }^{16}$ This randomized controlled trial was performed at the beginning of the main surgeon's training period. The authors speculate that the learning curve of the leading surgeon most likely contributed to the laparoscopic procedure's complication rate. Some researchers have already estimated that a minimum of 40 laparoscopic procedures is required to avoid the influence of the learning curve. ${ }^{17}$ So far, there has only been 1 research group, originating from Korea, which has published its research by conducting its study on a relatively large patient population (number of patients enrolled in both procedures was $>90$ ). However, the Korean researchers think one of the limitations of their study was its retrospective nature. The reason was that the use of laparoscopic or open method surgery was determined by the surgeon without a clarified selection criteria, and so bias may have been introduced. ${ }^{18}$ Other reviewers also questioned the frequency of certain complications or metastases that were often observed much more in open procedure. The difficult cases were treated by using the more familiar open methods and easy early-stage cancer cases were selected to be treated by the novel "new toy". ${ }^{19}$ There is inherent bias relating to case selection in retrospective studies. It is necessary to have 1 study with a large sample size without a selection bias to compare the 2 procedures fairly. That being said, the authors' special retrospective study was conducted with more than 400 patients involved in laparoscopic procedure, trying to avoid the selection bias. In Union Hospital, the authors have performed LRH since May 2008. Before May 2008, the Union Hospital only used ORH for the patients. After 2008, the Union Hospital has only used LRH and not ORH in all consecutive patients who needed surgical treatment for early-stage cervical cancer. In this retrospective study, there was no selection problem for different surgical techniques; the choice of technique depended solely on the time of treatment (before or after May 2008). The aim of this study was to compare clinical outcomes between LRH and ORH in early-stage cervical cancer.

\section{Materials and methods Patients}

A retrospective review of the medical records was performed for patients with FIGO (International Federation of
Gynecology and Obstetrics) stage IA (IA1, IA2), IB (IB1, IB2), and IIA (IIA1, IIA2) cervical cancer who underwent LRH from May 2008 to December 2013 or ORH from December 2000 to April 2008 at the Department of Obstetrics and Gynecology, Union Hospital, Tongji Medical College, Huazhong University of Science and Technology (Union Hospital, Wuhan, China). From May 2008, LRH was planned for all operable cervical cancer patients treated in the department. Collection and archiving of patient data was performed with written informed consent, and the study was approved by the hospital (Union Hospital, Wuhan, China) ethics committee.

The diagnosis of cervical cancer was histologically confirmed in all patients before surgery. In addition to this, the pretreatment evaluation included physical examination, vaginal/pelvic examination, chest X-ray, and pelvic magnetic resonance imaging. Cystoscopy and/or proctoscopy with biopsy were performed in patients with suspicious bladder/ bowel involvement. Positron emission tomography scan was recommended in case of suspicious distant metastasis. Moreover, adequate performance status and adequate functions of important internal organs were assessed, such as renal, hepatic, cardiac, and pulmonary functions, which were essential for surgery. All patients were informed of the risks and possible complications of surgery.

\section{Patient management}

All patients were operated on by the team of Professor Zehua Wang, consisting of 8 gynecologic-oncologists. The technique of ORH had been based on routine practice. The technique of LRH has been described previously. ${ }^{20,21} \mathrm{~A}$ harmonic scalpel was the main instrument for the dissection, division, and maintenance of hemostasis of all major surgical pedicles in the Obstetrics and Gynecology Department, and LRH was performed according to the C2 type of the QuerleuMorrow classification. ${ }^{22}$

In all patients, the urine catheter was removed 10 days after surgery and the bladder function was assessed by performing post-void residual catheterizations. In patients with a residual urine volume higher than $100 \mathrm{~mL}$, the bladder was recatheterized until normal voiding resumed. Adjuvant therapy was given in 2 circumstances: 1) patients with lymph node metastasis, parametrial involvement, or positive surgical margins, and 2) patients with 2 or more intermediate risk factors (ie, lymphovascular space involvement, deep stromal invasion, and tumor size $\geq 2 \mathrm{~cm}$ ). The adjuvant radiotherapy was performed in the cancer center of Union Hospital. Following surgery, patients underwent follow-up 
examinations every 3 months for 2 years, every 6 months in the third year, and once a year thereafter. The contents of the follow-up examinations included a gynecological examination; vaginal stump brushing cytological examination; ultrasound examinations for the pelvis, kidneys, ureters, and bladder; and, finally, a chest X-ray.

\section{Data collection and definitions}

Medical records were reviewed to collect and interpret data regarding disease and therapy. The following parameters were recorded: age, body mass index, FIGO stage, tumor size (diameter of the largest dimension of the primary tumor), histopathologic subtype, tumor grade, and involvement of lymph node and parametrium. Intra- and perioperative parameters included the following: operative time, estimated blood loss, lymph node count, status of the surgical margins, intraoperative complications, and early postoperative complications.

The operative time was measured from the first incision to the final suture. The blood loss was measured as the sum of the suctioned fluids and the weight of the sponges minus the use of irrigation fluids at the completion of the surgery. Intraoperative complications were defined as injury to the intestines, bladder, ureters, or great vessels. Early postoperative complications were defined as those that occurred within 30 days of surgery. Bladder dysfunction was diagnosed if a patient presented with a residual urine volume of more than $100 \mathrm{~mL}$ when the urine catheter was removed on the 10th postoperative day. Overall survival (OS) was defined as the time from surgery to death from any cause, or to the date of last contact. Progression-free survival was defined as the time from surgery to the first appearance of relapse, progression of existing disease, or to the date of last contact.

\section{Statistical analysis}

SPSS software (v.13.0) (SPSS Inc., Chicago, IL, USA) was used for statistical analysis. Fisher's exact test and Student's $t$-test were used to compare each group's clinical factors and values. The survival curves were produced by the Kaplan-Meier method by using the SPSS statistical software 13.0 (SPSS Inc.). All tests were 2-sided, and $p<0.05$ was considered statistically significant.

\section{Results}

According to the inclusion criteria, 412 patients underwent LRH and 139 patients underwent ORH for cervical cancer during the study period. Table 1 presents a comparison of the clinicopathologic factors in the LRH and ORH groups. The results demonstrated that there were no differences
Table I Comparison of clinicopathologic characteristics of patients $(n=551)$

\begin{tabular}{|c|c|c|c|}
\hline Characteristics & LRH (n=4 I 2) & ORH (n=139) & $p$-value ${ }^{a}$ \\
\hline Age & & & 0.23 \\
\hline $\begin{array}{l}\text { Median (range), } \\
\text { years }\end{array}$ & $44.19(25-76)$ & $40.52(23-62)$ & \\
\hline BMI & & & 0.16 \\
\hline $\begin{array}{l}\text { Median (range), } \\
\mathrm{kg} / \mathrm{m}^{2}\end{array}$ & $22.81(\mid 4.33-35.61)$ & $23.19(13.88-36.63)$ & \\
\hline \multicolumn{3}{|c|}{ Previous abdominal surgery, $\mathrm{n}(\%)$} & 0.13 \\
\hline 0 & $310(75.24)$ & III (79.86) & \\
\hline I & $95(23.06)$ & $26(|8.7|)$ & \\
\hline$\geq 2$ & $7(1.70)$ & $2(1.43)$ & \\
\hline FIGO stage, n (\%) & & & 0.32 \\
\hline la & $35(8.50)$ & $12(8.63)$ & \\
\hline $\mathrm{lb}$ & $331(80.34)$ & $105(75.54)$ & \\
\hline Ila & $46(11.16)$ & $22(15.83)$ & \\
\hline \multicolumn{3}{|c|}{ Histology of tumor, n (\%) } & 0.25 \\
\hline Squamous & $340(82.52)$ & $110(79.14)$ & \\
\hline Nonsquamous ${ }^{\mathrm{b}}$ & $72(17.48)$ & $29(20.86)$ & \\
\hline \multicolumn{3}{|c|}{ Histological grading, n (\%) } & 0.11 \\
\hline $\begin{array}{l}\text { Well } \\
\text { differentiated }\end{array}$ & $120(29.13)$ & $38(27.34)$ & \\
\hline $\begin{array}{l}\text { Moderately } \\
\text { differentiated }\end{array}$ & 166 (40.29) & $58(41.73)$ & \\
\hline $\begin{array}{l}\text { Poorly } \\
\text { differentiated }\end{array}$ & $92(22.33)$ & $30(21.58)$ & \\
\hline Unknown & $34(8.25)$ & $13(9.35)$ & \\
\hline $\begin{array}{l}\text { Positive lymph } \\
\text { nodes }\end{array}$ & & & 0.09 \\
\hline No & $359(87.14)$ & $119(85.61)$ & \\
\hline Yes & $53(12.86)$ & $20(14.39)$ & \\
\hline
\end{tabular}

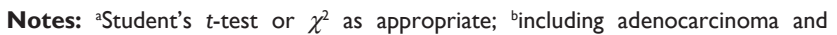
adenosquamous carcinoma.

Abbreviations: BMI, body mass index; LRH, laparoscopic radical hysterectomy with pelvic lymphadenectomy; ORH, open radical hysterectomy with pelvic lymphadenectomy; FIGO, International Federation of Obstetrics and Gynecology.

between the 2 groups with regard to age, body mass index, tumor size, grade, and positive lymph nodes. Table 2 demonstrates a comparison of surgical outcomes in the LRH and ORH groups. There were no differences in operating time. Estimated blood loss and transfusion requirements were

Table 2 Comparison of surgical outcomes $(n=55 \mathrm{I})$

\begin{tabular}{|c|c|c|c|}
\hline Characteristics & LRH $(n=4 \mid 2)$ & ORH $(n=139)$ & $p$-value \\
\hline Operating time & & & 0.01 \\
\hline Mean (range), min & $238.17(110-480)$ & $258.94(I 80-500)$ & \\
\hline Blood loss & & & $<0.01$ \\
\hline Mean (range), mL & $292.78(20-1,200)$ & $493.89(100-2,000)$ & \\
\hline \multicolumn{2}{|c|}{ Pelvic lymph nodes retrieved } & & $<0.01$ \\
\hline Mean (range), $\mathrm{n}$ & $24.35(8-66)$ & $20.24(9-44)$ & \\
\hline \multicolumn{2}{|c|}{ Postoperative hospital stay } & & $<0.01$ \\
\hline Mean (range), days & $12.16(7-21)$ & $15.35(9-33)$ & \\
\hline
\end{tabular}

Note: aStudent's $t$-test or $\chi^{2}$ as appropriate.

Abbreviations: LRH, laparoscopic radical hysterectomy with pelvic lymphadenectomy; $\mathrm{ORH}$, open radical hysterectomy with pelvic lymphadenectomy. 
Table 3 Comparison of perioperative complications $(n=551)$

\begin{tabular}{|c|c|c|c|}
\hline Complications & $\begin{array}{l}\text { LRH } \\
(n=4 \mid 2), \\
n(\%)\end{array}$ & $\begin{array}{l}\text { ORH } \\
(n=139), \\
n(\%)\end{array}$ & $p$-value ${ }^{a}$ \\
\hline Intraoperative complication & $7(1.70)$ & $2(1.44)$ & 0.22 \\
\hline Bladder injury & 1 & & \\
\hline Ureter injury & 2 & I & \\
\hline Vessel injury & 3 & 1 & \\
\hline Obturator nerve injury & I & & \\
\hline Postoperative complication & $36(8.74)$ & $10(7.19)$ & 0.14 \\
\hline Vaginal cuff infection & I & I & \\
\hline Hydronephrosis & 12 & 2 & \\
\hline Deep vein thrombosis & 5 & 2 & \\
\hline Urinary tract fistula formation & 3 & & \\
\hline Lymphocyst & 11 & 2 & \\
\hline Thrombophlebitis & 2 & & \\
\hline Bowel obstruction & 2 & 3 & \\
\hline Postoperative retention of urine & $149(36.17)$ & $32(23.02)$ & $<0.01$ \\
\hline
\end{tabular}

Note: aStudent's $t$-test or $\chi^{2}$ as appropriate.

Abbreviations: LRH, laparoscopic radical hysterectomy with pelvic lymphadenectomy; $\mathrm{ORH}$, open radical hysterectomy with pelvic lymphadenectomy.

significantly lower in the LRH group. Postoperative hospital stay was significantly shorter in the LRH group. A significant difference was found in the number of pelvic lymph nodes retrieved between the LRH and ORH groups. Table 3 demonstrates a comparison of perioperative complications in the LRH and ORH groups. The incidence of intraoperative complications was greater in the LRH group, but there was no observable significance. There was no significant difference found between the 2 groups when the total postoperative complications were compared. None of the patients required reoperation due to postoperative complications. In particular, hydronephrosis and lymphocyst were more frequent in the LRH group, while bowel obstruction was more frequent in the ORH group. Deep vein thrombosis was similarly present in LRH patients compared to the ORH patients.

After a median follow-up of 39 months (range 11-170 months), there were 10 and 16 cases lost to follow-up in laparoscopy and laparotomy group, respectively. Excluding the lost cases, 30 patients in the LRH group and 15 in the ORH group had recurrent disease and 24 patients in the LRH group and 13 in the ORH group died of the disease. The progression-free survival was $92.3 \%$ for the LRH group and $88.5 \%$ for the ORH group ( $p=0.290)$, and the OS was $92.7 \%$ for the LRH group and $90.7 \%$ for the ORH group ( $p=0.492)$ (Figure 1).

\section{Discussion}

For the aforementioned procedures, the present retrospective study appears to contain the largest relative number of subjects in regard to study size. The results suggest that estimated blood loss and transfusion requirements were significantly lower in the LRH group. Postoperative hospital stay was also significantly lower in the LRH group. The authors observed that the number of pelvic lymph nodes retrieved between the LRH and ORH group were significantly different. However, the operating time and perioperative complications between the LRH and ORH group showed no dissimilarity. The duration of the LRH operations varied widely (Table 2). Some of the authors have demonstrated a decrease in operating time as their experience increases; however, most of surgeons are still in the "early phase" of the learning curve. ${ }^{16,23,24}$ Nevertheless, although operative time decreased with surgeon experience and when the learning curve had been surpassed, the results of all studies are consistent with a longer duration of surgery for the laparoscopic approach. The authors' study supports the theory that surgical time in laparoscopy was significantly longer than laparotomy. The surgical time in the laparoscopic group was significantly longer as was shown in the metaanalysis of Wang et al. ${ }^{25}$ It has to be considered that the laparoscopic technique involves several steps such as extraction of lymph nodes using a bag device and insertion of a uterine manipulator and other laparoscopic instruments, all of which increase the overall skin-to-skin operative time. This study shows that there was less blood loss with laparoscopy when compare to the laparotomy. This finding was consistently reported in most studies. The laparoscopic groups showed less blood loss than the open group had demonstrated, as was evident in the meta-analysis. ${ }^{25}$ Blood loss at laparoscopy is less due to better visualization of the small vessels via magnification of the laparoscopic optical systems, which enables careful hemostasis of the operative field. ${ }^{24}$ Laparoscopic surgery is usually associated with a short hospital stay dues to its the advantages which include early mobilization and less intense pain. Thus far, only 1 single-center, randomized, controlled trial enrolling 30 cervical cancer patients has been done to study the postoperative pain after laparoscopic radical hysterectomy and abdominal hysterectomy. ${ }^{16}$ The trial results show that laparoscopic radical hysterectomy provided lower pain scores after 36 hours of observation in this series. In a study by $\mathrm{Li}$ et $\mathrm{al},{ }^{23}$ bowel recovery time of the laparoscopy group (1.96 days) was shorter than the open surgery group (2.4 days). The results demonstrate that postoperative hospital stay was significantly shorter in the LRH group. Differences in health care delivery systems and culture may account for the large variation in average hospital stay. In the study, the length of postoperative hospital stay was longer than in the Western countries, which was 

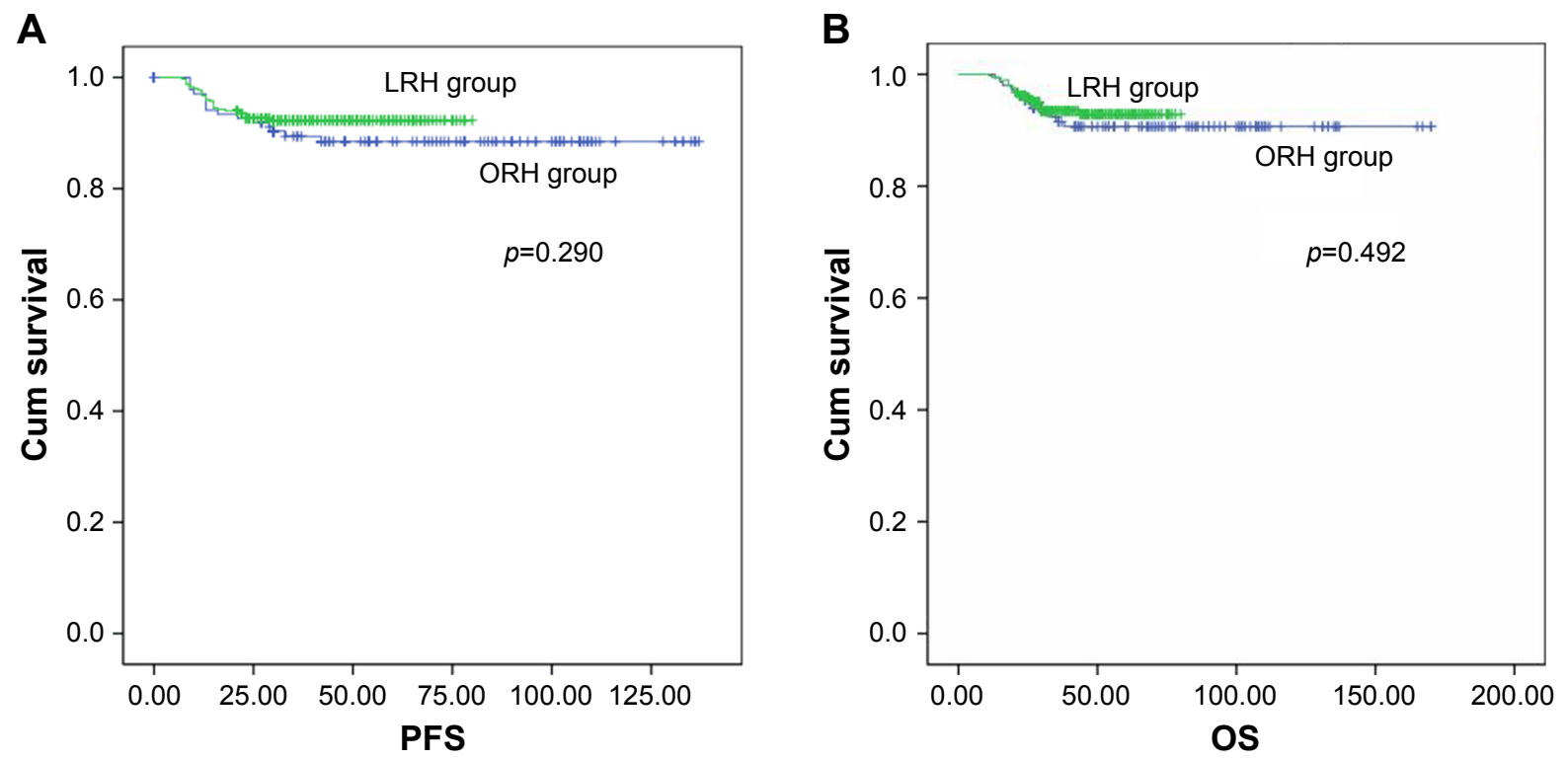

Figure I Kaplan-Meier curves for comparison of survival between groups.

Notes: Log-rank test was used to calculate statistical significance. There was no significant difference in PFS (A) and OS (B) between the LRH and the ORH groups. Abbreviations: LRH, laparoscopic radical hysterectomy with pelvic lymphadenectomy; PFS, progression-free survival; ORH, open radical hysterectomy with pelvic lymphadenectomy; OS, overall survival; Cum, cumulative.

similar to what was seen in Korea in Asia, ${ }^{26}$ which could be accounted for by differences in health care delivery and cultural variations. Other factors to take into regard include that Chinese patients prefer to remain in the hospital postoperatively for a considerable period of time, despite doctors' medical advice regarding earlier discharge; for example, a length of stay of 13.81 days post-LRH was seen in the study by Li et al. ${ }^{23}$ In addition to that, insurance companies may be less concerned about the duration of hospital stay in regard to patients with cancer. As the most common long-term complication after radical hysterectomy for cervical cancer, the incidence of postoperative bladder dysfunction has been reported to range from $16.3 \%$ to $76 \% .{ }^{23,26,27}$ In this study, the authors found bladder dysfunction was the most common early postoperative complication, occurring in $36.17 \%$ of the LRH group. Most of the patients resumed normal voiding within 30 days of surgery. The only single-center randomized controlled trial also compared the perioperative outcomes after laparoscopic radical hysterectomy and abdominal radical hysterectomy. The trial results demonstrated that the perioperative and serious postoperative complication ratios were comparable between the groups, but survival results were not available. ${ }^{16}$ The current results demonstrate that there was no significant difference observed between the 2 groups in this context. As our study is a surgical series comparing the immediate surgical and survival outcomes between LRH and ORH, we estimated only the intraoperative and postoperative complications occurring within 60 days following the surgery. LRH and ORH. Therefore, the genitourinary complications reported in the study are complications associated with the surgery itself, but the incidence of genitourinary complications was very low. The authors believe that estimating the long-term complications may not be totally accurate due to the retrospective nature of the study. There was no significant difference between the LRH and ORH groups, as was illustrated in the meta-analysis. ${ }^{25}$ In most studies, there were no differences in the rate of intraoperative complications observed when comparing between laparoscopy and open surgery. Some studies even demonstrated a trend toward a higher number of complications in patients who underwent laparotomy. Nam et a ${ }^{27}$ studied long-term survival outcomes in a matched cohort study between patients who underwent laparoscopic and open radical hysterectomy in early-stage cervical cancer. There were no statistically significant differences between recurrence-free survival and disease-specific survival between LRH and ORH groups. Thus far, definitive data about differences in OS, disease-free survival, and recurrence between laparoscopy and conventional open surgery has not been found. Li et al ${ }^{23}$ speculate that only a randomized study comparing the 2 groups would provide quintessential evidence of equality. In this study, certain limitations were present. First, there might be hidden biases, especially in series where older series are compared to a more recent one. Second, this was a retrospective study; therefore, the information about the long-term complications may not be completely accurate. 


\section{Conclusion}

On the basis of the present data, LRH can be considered as a safe and effective alternative to conventional open surgery for early-stage cervical cancer. Despite some potential disadvantages, the procedure has distinct advantages and similar efficacy in comparison to ORH. Furthermore, multicenter randomized clinical trials with a longer follow-up should be performed to confirm the overall oncologic outcomes of laparoscopic procedure.

\section{Acknowledgment}

This work was supported by National Natural Science Foundation of China (81202058, 81272860, and 81472443).

\section{Disclosure}

The authors report no conflicts of interest in this work.

\section{References}

1. Parkin DM, Bray F, Ferlay J, Pisani P. Estimating the world cancer burden: Globocan 2000. Int J Cancer. 2001;94(2):153-156.

2. Pecorelli S. Revised FIGO staging for carcinoma of the vulva, cervix, and endometrium. Int J Gynecol Obstet. 2009;105(2):103-104.

3. Averette HE, Nguyen HN, Donato DM, et al. Radical hysterectomy for invasive cervical cancer: a 25 -year prospective experience with the Miami technique. Cancer. 1993;71(Suppl 4):1422-1437.

4. Underwood PB Jr, Wilson WC, Kreutner A, Miller MC 3rd, Murphy E. Radical hysterectomy: a critical review of twenty-two years' experience. Am J Obstet Gynecol. 1979;134(8):889-898.

5. Comerci G, Bolger BS, Flannelly G, Maini M, de Barros Lopes A, Monaghan JM. Prognostic factors in surgically treated stage IB-IIB carcinoma of the cervix with negative lymph nodes. Int $J$ Gynecol Cancer. 1998;8(1):23-26.

6. Ayhan A, Tuncer ZS, Yarali H. Complications of radical hysterectomy in women with early stage cervical cancer: clinical analysis of 270 cases. Eur J Surg Oncol. 1991;17(5):492-494.

7. Cuschieri A. Technology for minimal access surgery. Interview by Judy Jones. BMJ. 1999;319(7220):1304.

8. Reich H, DeCaprio J, McGlynn F. Laparoscopic hysterectomy. J Gynecol Surg. 1989;5(2):21-36.

9. Querleu D. [Radical hysterectomies by the Schauta-Amreich and Schauta-Stoeckel techniques assisted by celioscopy]. J Gynecol Obstet Biol Reprod. 1991;20(5):747-748. French.

10. Querleu D. Laparoscopically assisted radical vaginal hysterectomy. Gynecol Oncol. 1993;51(2):248-254.

11. Nezhat CR, Burrell MO, Nezhat FR, Benigno BB, Welander CE. Laparoscopic radical hysterectomy with paraaortic and pelvic node dissection. Am J Obstet Gynecol. 1992;166(3):864-865.
12. Canis M, Mage G, Pouly JL, et al. Laparoscopic radical hysterectomy for cervical cancer. Baillieres Clin Obstet Gynecol. 1995;9(4):675-689.

13. Hsieh YY, Lin WC, Chang CC, Yeh LS, Hsu TY, Tsai H. Laparoscopic radical hysterectomy with low paraaortic, subaortic and pelvic lymphadenectomy: results of short-term follow-up. J Reprod Med. 1998; 43(6):528-534.

14. Kim DH, Moon JS. Laparoscopic radical hysterectomy with pelvic lymphadenectomy for early, invasive cervical carcinoma. J Am Assoc Gynecol Laparosc. 1998;5(4):411-417.

15. Spirtos NM, Schlaerth JB, Kimball RE, Leiphart VM, Ballon SC. Laparoscopic radical hysterectomy (type III) with aortic and pelvic lymphadenectomy. Am J Obstet Gynecol. 1996;174(6):1763-1768.

16. Campos LS, Limberger LF, Stein AT, Kalil AN. Postoperative pain and perioperative outcomes after laparoscopic radical hysterectomy and abdominal radical hysterectomy in patients with early cervical cancer: a randomised controlled trial. Trials. 2013;14:293.

17. Hwang JH, Yoo HJ, Joo J, et al. Learning curve analysis of laparoscopic radical hysterectomy and lymph node dissection in early cervical cancer. Eur J Obstet Gynecol Reprod Biol. 2012;163(2):219-223.

18. Park JY, Kim DY, Kim JH, Kim YM, Kim YT, Nam JH. Laparoscopic versus open radical hysterectomy in patients with stage IB2 and IIA2 cervical cancer. J Surg Oncol. 2013;108(1):63-69.

19. Pergialiotis V, Rodolakis A, Christakis D, Thomakos N, Vlachos G, Antsaklis A. Laparoscopically assisted vaginal radical hysterectomy: systematic review of the literature. J Minim Invasive Gynecol. 2013; 20(6):745-753.

20. Shen $Y$, Wang Z. Total laparoscopic radical hysterectomy for treatment of uterine malignant tumors: analysis of short-term therapeutic efficacy. J Huazhong Univ Sci Technolog Med Sci. 2010;30(3):375-378.

21. Yang L, Cai J, Dong W, et al. Laparoscopic radical hysterectomy and pelvic lymphadenectomy can be routinely used for treatment of earlystage cervical cancer: a single institute experience with 404 patients. J Minim Invasive Gynecol. 2015;22(2):199-204.

22. Querleu D, Morrow CP. Classification of radical hysterectomy. Lancet Oncol. 2008;9(3):297-303.

23. Li G, Yan X, Shang H, Wang G, Chen L, Han Y. A comparison of laparoscopic radical hysterectomy and pelvis lymphadenectomy and laparotomy in the treatment of Ib-IIa cervical cancer. Gynecol Oncol. 2007;105(1):176-180.

24. Frumovitz M, dos Reis R, Sun CC. Comparison of total laparoscopic and abdominal radical hysterectomy for patients with early-stage cervical cancer. Obstet Gynecol. 2007;110(1):96-102.

25. Wang YZ, Deng Li, Xu HC, Zhang Y, Liang ZQ. Laparoscopy versus laparotomy for the management of early stage cervical cancer. $B M C$ Cancer. 2015;15:928.

26. Lee EJ, Kang H, Kim DH. A comparative study of laparoscopic radical hysterectomy with radical abdominal hysterectomy for early-stage cervical cancer: a long-term follow-up study. Eur J Obstet Gynecol Reprod Bio. 2011;156(1):83-86.

27. Nam JH, Park JY, Kim DY, Kim JH, Kim YM, Kim YT. Laparoscopic versus open radical hysterectomy in early-stage cervical cancer: long-term survival outcomes in a matched cohort study. Ann Oncol. 2012;23(4): 903-911.
OncoTargets and Therapy

\section{Publish your work in this journal}

OncoTargets and Therapy is an international, peer-reviewed, open access journal focusing on the pathological basis of all cancers, potential targets for therapy and treatment protocols employed to improve the management of cancer patients. The journal also focuses on the impact of management programs and new therapeutic agents and protocols on

\section{Dovepress}

patient perspectives such as quality of life, adherence and satisfaction. The manuscript management system is completely online and includes a very quick and fair peer-review system, which is all easy to use. Visit http://www.dovepress.com/testimonials.php to read real quotes from published authors. 